\title{
Compulsory vaccination: case isolation is likely to be more effective
}

An editorial error occurred in this letter by Mark Temple (BMJ 2019;366:14472; doi:10.1136/bmj.14472).

In the third paragraph the "red book" referred to is the 1988 publication from the World Health Organization, not the Personal Child Health Record as published.
The last sentence of the third paragraph should have read: The World Health Organization publication known as the "red book" also states that the removal of endemicity was due to UK local authorities reacting "effectively with surveillance, the isolation of cases, and the vaccination of contacts" (page 326). ${ }^{3}$

This has been corrected online. 